\title{
INTERVENCIÓN PSICOLÓGICA A CANDIDATOS DE CIRUGÍA BARIÁTRICA EN UN HOSPITAL PÚBLICO DE BRASIL
}

\author{
Marinna Mensorio ${ }^{1,2, a}$, Áderson Costa-Júnior 3 ,b
}

\begin{abstract}
RESUMEN
Se investigó un programa de intervención psicológica a pacientes candidatos a cirugía bariátrica, evaluando estrategias de afrontamiento adoptadas, manifestaciones de ansiedad y cambios de estilo de vida, comparándolos a un grupo control. El programa cognitivo-conductual consiste en doce sesiones de grupo en el que se abordan cuestiones relacionadas con el proceso de cambio de hábitos y la preparación para la cirugía. La comparación entre las evaluaciones pre y posintervención y entre grupos mostró tendencias de mejoría en el grupo experimental; sin embargo, las variables de ansiedad empeoraron en algunos pacientes. Se destaca la importancia del tratamiento psicológico continuo como promotor de las habilidades necesarias para hacer frente a situaciones adversas de la obesidad y del tratamiento. Se sugiere para futuras intervenciones estudios con muestras más grandes, y mayor atención a variables de ansiedad.
\end{abstract}

Palabras clave: Obesidad; Cirugía bariátrica; Ansiedad; Estrategias de afrontamiento (Fuente: DeCS BIREME).

\section{PSYCHOLOGICAL INTERVENTION TO CANDIDATES FOR SURGERY BARIATRIC IN A PUBLIC HOSPITAL IN BRAZIL}

\begin{abstract}
We evaluated a psychological intervention program to patients eligible for bariatric surgery, performing lifting coping strategies, anxiety manifestations and lifestyle changes, comparing them to obese patients without exposure to the intervention. The cognitive-behavioral program consists of 12 group sessions, in which they are addressed issues relevant to the process of habit change and preparation for surgery. The comparison of pre and post-intervention and between groups showed improvements in the experimental group. It is highlighted the importance of continued counseling as a promoter of skills required to face adverse situations of obesity and treatment. Studies with larger samples, and more attention to anxiety variables are suggested.
\end{abstract}

Keywords: Obesity; Bariatric surgery; Anxiety; Coping strategies (Source: MeSH NLM).

\section{INTRODUCCIÓN}

La obesidad $\left(\mathrm{IMC}>30 \mathrm{~kg} / \mathrm{m}^{2}\right)$ es considerada una de las epidemias evitables más grandes en el mundo occidental (1-3). Datos de la Organización Mundial de la Salud (OMS) ${ }^{(4)}$ indican la creciente tendencia de la obesidad, de manera que la incidencia en todo el mundo se ha más que duplicado desde 1980, pues en 2008 más de 1,4 mil millones de adultos (de 20 o más años de edad) tenía sobrepeso. La obesidad se encuentra relacionada con una mayor incidencia de otras enfermedades, entre las cuales están las enfermedades cardíacas y psicológicas como la ansiedad y la depresión ${ }^{(5,6)}$.

Para su tratamiento y control, los cambios en el estilo de vida se presentan como pilares fundamentales.
Según Gade ${ }^{(7)}$, Travado et al. ${ }^{(8)}$ y White ${ }^{(9)}$, un enfoque biopsicosocial y las contribuciones teóricas y filosóficas de la perspectiva cognitivo-conductual son fundamentales para el éxito potencial del tratamiento. Por otro lado, para las personas con un índice de masa corporal (IMC) de más de 40 , o mayores de 35 con comorbilidades, la cirugía bariátrica ha sido considerada como el mejor tratamiento médico, teniendo en cuenta los criterios de la pérdida y mantenimiento del peso (10), así como la reversión de los efectos adversos de la obesidad ${ }^{(11)}$.

Sin embargo, entre el 20 a $30 \%$ de los pacientes sometidos a cirugía comienzan a recuperar peso después de 24 meses (promedio) ${ }^{(10)}$, poniéndose de manifiesto la necesidad de intervenciones psicosociales

\footnotetext{
CAPES Foundation, Ministry of Education of Brasil. Brasília, Brasil.

Facultad de Psicología, Universitat de Valéncia. Valencia, España.

Instituto de Psicologia, Universidade de Brasília. Brasília, Brasil.

a. Psicóloga, maestra en Psicología; ${ }^{\text {b }}$ psicólogo, doctor en Psicología

Este estudio es parte de la disertación de maestría: Mensorio, MS \& Costa-Jr, AL. Análise de estratégias de enfrentamento, ansiedade e hábitos em pacientes elegíveis à cirurgia bariátrica, com e sem acompanhamento psicológico. Dissertação de Mestrado. Brasília: Insituto de Psicologia, UnB, 2013.

Recibido: 07-09-15 Aprobado: 09-03-16
}

Citar como: Mensorio M, Costa-Júnior A. Intervención psicológica a candidatos de cirugía bariátrica en un hospital público de Brasil. Rev Peru Med Exp Salud Publica. 2016;33(1):120-7. doi: 10.17843/rpmesp.2016.331.1941 
que mejoren las condiciones psicológicas del paciente para mantener nuevos hábitos alimentarios ${ }^{(12)}$.

Una de las variables funcionalmente relacionadas con el éxito potencial del tratamiento es la manera en la que el paciente afronta la condición de la obesidad, incluyendo necesidades, limitaciones y cambios en los hábitos ${ }^{(13,14)}$. El afrontamiento, o coping, se refiere a las estrategias adoptadas por las personas para adaptarse a circunstancias estresantes, negativas o desagradables, y se define como un conjunto de esfuerzos cognitivos y de comportamiento utilizados por los individuos con el fin de hacer frente a las demandas específicas que pueden surgir en situaciones de estrés ${ }^{(15,16)}$. Las estrategias pueden ser enfocadas en el problema (el cambio de hábitos para que pueda bajar de peso, por ejemplo), o la emoción (lidiar con sentimientos relacionados con condición de obesidad, por ejemplo).

Conradt et al. (17) señalaron que las situaciones típicas en las que los pacientes obesos requieren estrategias de afrontamiento eficaces incluyen evaluaciones negativas de sí mismos o de otros, la adherencia al ejercicio físico regular, y la exposición a situaciones de riesgo ambientales (tiendas de ropa y fiestas sociales). Puhl y Brownell (18) resaltan la necesidad de realizar más estudios sobre la eficacia de los métodos de afrontamiento de eventos considerados potencialmente adversos o estresantes del propio tratamiento de la obesidad, tales como los cambios en los hábitos alimentarios, la participación en la actividad física regular, y las intervenciones quirúrgicas.

Oliveira et al. (19) resaltan que el papel del psicólogo es evaluar si el individuo está preparado emocionalmente para la cirugía, y ayudarlo en la comprensión de los aspectos resultantes del proceso pre y posquirúrgico. Sin embargo, mientras que las evaluaciones psicológicas prequirúrgicas son comunes entre los pacientes que pueden beneficiarse de la cirugía bariátrica, el valor de estas evaluaciones como predictores de la pérdida de peso exitosa es todavía cuestionado ${ }^{(20)}$. Por lo tanto, se destaca la necesidad de investigaciones sobre la eficacia de los servicios que desarrollan preparativos psicológicos prequirúrgicos, con el fin de comprender su verdadero propósito como un facilitador de estrategias de cambio de hábitos.

Este estudio tiene como objetivo evaluar las estrategias de afrontamiento, el nivel de ansiedad y cambio de estilo de vida de pacientes obesos asistidos en un hospital público de la ciudad de Brasilia en Brasil, participantes de un programa de preparación psicológica para cirugía bariátrica, comparando los resultados con un grupo control de pacientes sin este tratamiento, con el fin de subvencionar el desarrollo de intervenciones de salud más eficaces y humanizadas.

\section{MATERIALES Y MÉTODOS}

\section{DISEÑO Y POBLACIÓN DE ESTUDIO}

Se diseñó un estudio cuasiexperimental, implementando un programa de preparación psicológica para la cirugía bariátrica. Los participantes fueron 40 pacientes divididos en dos grupos: grupo experimental (GE), $n=15$, y grupo control (GC), n=25. Los pacientes eran adultos en lista de espera para la cirugía, y fueron asignados a dos grupos de acuerdo con su situación en la lista de espera (pacientes al principio y al final de la lista).

El GE inicial consistió en quince pacientes invitados a participar en el acompañamiento psicológico de preparación para la cirugía bariátrica, aunque solo ocho finalizaron la intervención. Las razones mencionadas para el abandono fueron problemas familiares o financieros y falta de interés. El grupo experimental final estaba compuesto por ocho pacientes, con media de edad de 40,30 años (DT=12,37), de los cuales siete eran mujeres, y con un nivel sociocultural medio.

En paralelo, para constituir el GC, 25 pacientes fueron contactados por teléfono e invitados a participar en el estudio, seleccionados por orden de la lista de espera para cirugía bariátrica. Al final, solo diez pacientes hicieron las dos etapas de evaluación. El grupo tenía siete participantes mujeres, con una media de edad de 49,4 años (DE=12,7), con nivel sociocultural medio.

\section{CRITERIOS DE INCLUSIÓN}

Pacientes adultos (entre 18-65 años), portadores de obesidad grado II o más (IMC>35), con comprensión de lectura y escrita (al menos escuela primaria incompleta) y sin enfermedades psicológicas graves.

\section{INSTRUMENTOS}

Cuestionario sociodemográfico: un cuestionario sociodemográfico creado ad-hoc para este estudio, que recogía datos sobre sexo, edad, altura, peso, antecedentes psiquiátricos, antecedentes de alcoholismo, estado civil, lugar de residencia; número de hijos, la educación y la profesión.

Entrevista semiestructurada: hecha específicamente para este estudio, compuesta por siete ítems, las preguntas buscaban recoger datos de afrontamiento de la situación de obesidad y cambio de hábitos.

Escala de modos de afrontamiento de problemas (EMEP; adaptación al portugués de Gimenes y Queiroz (21)). El instrumento evalúa las estrategias cognitivas de afrontamiento y/o de comportamiento adoptadas contra 
un factor estresante específico. La escala contiene 45 ítems, divididos en cuatro factores: afrontamiento centrado en el problema; afrontamiento enfocado en la emoción; afrontamiento religioso/espiritual, y la búsqueda de apoyo social.

Beck Anxiety Inventory (BAI, Beck, Epstein, Brown y Steer (22); traducido al portugués y adaptado a la población brasileña por Cunha $\left.{ }^{(23)}\right)$, es un instrumento autoadministrado de 21 items, que permite la investigación de la intensidad de las manifestaciones de la ansiedad. La suma de las puntuaciones obtenidas en cada elemento (escala Likert) genera una puntuación total entre $0-63$, que se divide en cuatro grados de intensidad de la manifestación de la ansiedad: mínima, leve, moderada y grave.

\section{PROCEDIMIENTO}

Los pacientes interesados en la cirugía bariátrica y que cumplen los requisitos, pasan a formar parte de una lista de espera (tiempo medio de espera 3-4 años). Se contacta con ellos telefónicamente 3 meses antes de la cirugía, momento en que se ofrece la participación en un grupo prequirúrgico bariátrico de asistencia psicológica (grupo experimental).

La intervención psicológica se basó en los principios teóricos y filosóficos de la Terapia Cognitivo-Conductual $(7,24)$ y de la Terapia de Grupo ${ }^{(25)}$ aplicados a contextos de obesidad. Consistía en un acompañamiento centrado en el cambio de hábitos y de estilos de vida necesarios para el éxito potencial pre y posquirúrgico, direccionándose a las variables funcionalmente relacionadas con el tratamiento de la obesidad. Se desarrolló a lo largo de 12 sesiones, consecutivas y semanales, con duración aproximada de 90 minutos, donde se abordaron temas específicos y previamente establecidos, considerados relevantes para el proceso de cambios de hábitos y preparación para la cirugía. Cada sesión de intervención tuvo la siguiente estructura:

A). Rompe hielos: saludos, acogida, y contacto inicial con el grupo, a través de la discusión de cuestiones de rutina, tales como: "¿cómo ha estado tu fin de semana?".

B). Análisis del registro alimentario y de las emociones: existen estudios que refuerzan la necesidad y relevancia de la autobservación y autoevaluación en el proceso de cambio de hábitos ${ }^{(26)}$ - así, se optó por solicitar a todos los participantes que rellenasen de manera diaria un registro alimentario y de emociones relacionadas al momento de comer y con conductas de cambio necesarias para el proceso de la cirugía. El registro contiene informaciones sobre: tiempo dedicado a cada comida; número de comidas diarias; sitio utilizado para comer y compañía en este momento (p.ej., en la mesa de casa, adelante del ordenador del trabajo, conduciendo; solo, con mi pareja, etc.); grado percibido de hambre antes y después de comer; picoteo entre comidas; tipo de alimento y cantidad; y sentimientos vivenciados antes y después de comer. El registro buscaba, entre otros objetivos, controlar el tiempo dedicado a masticar; controlar frecuencia que uno se alimenta (no "saltar" comidas); diferenciar el hambre y las ganas de comer; relación entre sentimiento y hora de comer. El investigador analizaba, con cada paciente del grupo, cómo estuvo su semana en términos de calidad de alimentos, adhesión a las orientaciones y la relación entre alimentos y las emociones. Hay una orientación hacia las necesidades de cada paciente, aunque que todos los demás miembros del grupo pueden escuchar y participar.

C). Discusión de los temas enfocados dentro de los objetivos de cada sesión. La estructura, temas y objetivos de cada sesión son presentados en la Tabla 1.

D). Establecimiento de metas para las próximas semanas: a partir de las aportaciones de los participantes sobre su evolución y del tema especificado para cada sesión, se establecían metas colectivas (e individuales cuando necesario), de cambio a corto y largo plazo, tales como: "masticar más lentamente", "mantener el autocontrol", "decir NO", etc.

Teniendo en cuenta el carácter de seguimiento previsto para este estudio, y el diseño cuasiexperimental, la aplicación de los instrumentos se produjo en dos momentos: antes del inicio de la primera sesión del GE y una semana después de la última sesión. Para el GC, los instrumentos se aplicaron en los mismos momentos. Además, se informa que los pacientes del GC han tenido igual acceso a la intervención posteriormente, respetando siempre su momento en la lista de espera.

\section{ANÁLISIS DE DATOS}

El análisis se realizó mediante comparaciones entre grupos (macroanálisis) y comparaciones específicas intrasujeto (microanálisis): para integrantes de un mismo grupo se utilizó la prueba de Wilcoxon y para la comparación entre grupos independientes el test de Mann-Whitney. Los datos cuantitativos fueron analizados con el programa SPSS Versión 19.0. Se realizaron análisis descriptivos y comparaciones de medias y medianas, utilizando pruebas no paramétricas teniendo en cuenta el tamaño de la muestra (teniendo en cuenta la naturaleza de la muestra, no se aplicaron pruebas paramétricas).

\section{CONSIDERACIONES ÉTICAS}

El proyecto de investigación fue aprobado por el Comité de Ética en Investigación de la Facultad de Salud, Universidad de Brasilia, con el número de protocolo CEP: 096/11. 
Tabla 1. Descripción de las doce sesiones aplicadas durante la intervención

\begin{tabular}{|c|c|c|c|}
\hline Sesión & Estrategias principales & Tema específico & Objetivos \\
\hline 1 & $\begin{array}{l}\text { Presentación del terapeuta de } \\
\text { grupo; breve explicación de lo que } \\
\text { es la terapia de grupo y terapia } \\
\text { cognitivo-conductual; presentación } \\
\text { de los miembros del grupo; entrega } \\
\text { del contrato terapéutico; entrega y } \\
\text { explicación del registro alimentario }\end{array}$ & $\begin{array}{l}\text { "Masticar; el sitio ideal para comer; } \\
\text { y las funciones de la comida en mi } \\
\text { vida". }\end{array}$ & $\begin{array}{l}\text { Psicoeducación: ofrecer orientaciones iniciales } \\
\text { sobre "cómo comer" y promover la reflexión } \\
\text { sobre "qué significa el alimento en mi vida". }\end{array}$ \\
\hline 2 & & $\begin{array}{l}\text { "Influencia del pensamiento y la } \\
\text { importancia de la familia en el } \\
\text { proceso de tratamiento". }\end{array}$ & $\begin{array}{l}\text { Discutir aspectos relacionados a la influencia } \\
\text { del pensamiento en la toma de decisión y la } \\
\text { importancia de agregar la familia al proceso de } \\
\text { cambio. }\end{array}$ \\
\hline 3 & & "La ansiedad y el hambre". & $\begin{array}{l}\text { Que el paciente empiece a observar la } \\
\text { relación que él establece entre emociones } \\
\text { (especialmente ansiedad) y el hecho de comer, } \\
\text { enseñando estrategias para empezar de } \\
\text { deshacer esta relación. }\end{array}$ \\
\hline 4 & & $\begin{array}{l}\text { "La autoobservación y los } \\
\text { atracones - comer por placer vs. } \\
\text { necesidad de comer, El hambre } \\
\text { frente a las ganas de comer". }\end{array}$ & $\begin{array}{l}\text { Que el paciente empiece a diferenciar el } \\
\text { hambre de las ganas de comer, observando } \\
\text { reacciones corporales y emocionales. }\end{array}$ \\
\hline 5 & & $\begin{array}{l}\text { "Cambio de hábitos, autocontrol y } \\
\text { la autodisciplina". }\end{array}$ & $\begin{array}{l}\text { Que el paciente, a partir del anterior } \\
\text { entendimiento de lo que es hambre y lo que son } \\
\text { ganas, pueda empezar a aplicar estrategias de } \\
\text { autocontrol útiles para el momento de comer } \\
\text { (o picotear) y empiece a establecer rutinas de } \\
\text { disciplina más controladas. }\end{array}$ \\
\hline 6 & & "Prácticas de relajación". & $\begin{array}{l}\text { Aplicar y enseñar técnica de relajación como } \\
\text { estrategia de manejo de ansiedad que pueda } \\
\text { ser utilizada por el paciente especialmente en } \\
\text { el momento posquirúrgico. }\end{array}$ \\
\hline 7 & Evaluación de la semana & $\begin{array}{l}\text { "El enfoque en peso, ¿se centran en } \\
\text { qué?". }\end{array}$ & $\begin{array}{l}\text { Generar ampliación del objetivo de pérdida de } \\
\text { peso- que vaya más allá del peso y se pueda } \\
\text { centrar en la búsqueda por salud y calidad de } \\
\text { vida. }\end{array}$ \\
\hline 8 & $\begin{array}{l}\text { Refuerzo positivo de los éxitos } \\
\text { Discusión de las dificultades } \\
\text { experimentadas }\end{array}$ & $\begin{array}{l}\text { "Los objetivos de la cirugía y } \\
\text { reestructuración de objetivos". }\end{array}$ & $\begin{array}{l}\text { Continuación del pensamiento del tema } \\
\text { anterior, que la cirugía no sea el resultado de } \\
\text { una búsqueda estética o de otros (familiares, } \\
\text { amigos), pero sí una búsqueda genuina } \\
\text { por calidad de vida, buscando estimular la } \\
\text { motivación intrínseca para el cambio. }\end{array}$ \\
\hline 9 & Discusión de tema específico & $\begin{array}{l}\text { “Cómo me veo a mí mismo y } \\
\text { como me ven los demás". }\end{array}$ & $\begin{array}{l}\text { Generar discusión sobre autoobservación e } \\
\text { influencia de opiniones de los demás en mi vida. }\end{array}$ \\
\hline 10 & & "La asertividad". & $\begin{array}{l}\text { Definir asertividad y aplicar los conceptos a la } \\
\text { vida diaria. }\end{array}$ \\
\hline 11 & & $\begin{array}{l}\text { "La autoestima, la autoimagen y } \\
\text { la imagen corporal". }\end{array}$ & $\begin{array}{l}\text { Objetivo: Desarrollar estrategias para lidiar } \\
\text { con el cambio del cuerpo y mantenimiento de } \\
\text { autoestima saludable. }\end{array}$ \\
\hline 12 & & $\begin{array}{l}\text { "El posquirúrgico inmediato y } \\
\text { tardío" (sesión con los miembros } \\
\text { de la familia). }\end{array}$ & $\begin{array}{l}\text { Ofrecer orientaciones directas a paciente y } \\
\text { familiares, por medio de psicoeducación, sobre } \\
\text { el posquirúrgico inmediato - estrategias sobre } \\
\text { cómo lidiar con el posible dolor, con las ganas } \\
\text { de comer y con expectativas a corto plazo. }\end{array}$ \\
\hline
\end{tabular}

\section{RESULTADOS}

\section{GRUPO EXPERIMENTAL}

Los resultados de los análisis de las respuestas de los participantes a la EMEP, antes de la intervención psicológica (Fase 1), demostraron la priorización por las estrategias centradas en el problema (factor $1, M=3,96$ ) y en el afrontamiento religioso/fantasioso/ilusiones (factor $3, M=3,67$ ), seguido de la búsqueda de apoyo social (factor $4, M=2,72$ ) y enfoque en la emoción (factor 2, $M=2,59)$. En la segunda fase, se mantuvo el orden de los factores (problema, $M=4,13$; pensamientos religiosos/ ilusiones, $M=3,11$; búsqueda de apoyo social, $M=2,95$; y emoción, $M=2,28$ ). Sin embargo, hubo un aumento en las puntuaciones de las estrategias centradas en los problemas y la búsqueda de apoyo social. Análisis 
estadísticos (prueba de Wilcoxon) demostraron una disminución significativa $(p<0,05)$ en las puntuaciones de afrontamiento centrado en la emoción y en los pensamientos religiosos/ilusión. No hubo diferencias significativas en las puntuaciones de los factores foco en el problema y apoyo social (Tabla 2).

En cuanto a las puntuaciones generales de evaluación de la ansiedad, fueron observadas mejoras: en la primera fase cuatro pacientes obtuvieron puntuaciones mínimas de ansiedad; tres puntuaciones leves y uno con puntuación moderada; no se registraron puntuaciones graves. En la segunda fase, estos números fueron seis, uno y uno, respectivamente, ningún paciente con puntuación grave. Se observó, igualmente, reducción en las puntuaciones promedias de ansiedad $(\mathrm{M}=11$ antes de la intervención; $M=8,3$ después; $p=0,553 ; v=17,5)$.

\section{GRUPO CONTROL}

En la Fase 1 se observó priorización por las estrategias centradas en el problema $(M=3,595)$ y en el pensamiento religioso/ilusión $(M=3,156)$, seguido de la búsqueda de apoyo social $(M=2,707)$ y enfoque en la emoción $(M=2,306)$. En la Fase 2, la elección por tipo de estrategia siguió el mismo orden, aunque se observó una reducción en la media de afrontamiento para todos los factores (problema, $\mathrm{m}=3,494$; pensamientos religiosos/ilusiones, $\mathrm{m}=3,301$; apoyo social, $\mathrm{m}=2,56$; $\mathrm{y}$ emoción, $m=1,953 ; p>0,05)$, excepto para pensamiento religioso y fantasioso (Tabla 3 ).

En relación a las puntuaciones de ansiedad, en la Fase 1 se registraron tres pacientes con puntuaciones mínimas de ansiedad, tres con puntuaciones leves, tres con puntuaciones moderadas y uno con puntuación severa. En la Fase 2, había cuatro, cero, tres y tres, respectivamente (resultados no significativos: $p>0,05$ ).

\section{COMPARACIÓN DE GRUPOS}

Con respecto a la Fase 1, no se observaron diferencias significativas entre los dos grupos (Tabla 4). En la Fase 2 se registró diferencias significativas entre el GE y el GC con relación al factor 1 de la EMEP y la ansiedad (Tabla 5).

Tabla 2. Comparación de los valores de la EMEP por etapa, grupo experimental $(n=8)$

\begin{tabular}{|c|c|c|c|}
\hline Variable & Etapa 1 (pre) & Etapa 2 (post) & Valor $\mathbf{p}^{*}$ \\
\hline Factor 1 (afrontamiento centrado en el problema) & 4,06 & 4,02 & 0,326 \\
\hline Factor 2 (afrontamiento enfocado en la emoción) & 2,53 & 2,17 & 0,043 \\
\hline Factor 3 (afrontamiento religioso/espiritual) & 4,0 & 3,14 & 0,035 \\
\hline Factor 4 (búsqueda de apoyo social) & 2,8 & 2,9 & 0,232 \\
\hline
\end{tabular}

EMEP: Escala de modos de afrontamiento de problemas; *Test pareado de Wilcoxon

Tabla 3. Comparación de los valores de la EMEP por etapa, grupo control $(n=15)$

\begin{tabular}{lccc}
\hline Variable & Etapa 1 (pre) & Etapa 2 (post) & Valor $\mathbf{p}^{*}$ \\
\hline Factor 1 (afrontamiento centrado en el problema) & 3,55 & 3,44 & 0,683 \\
Factor 2 (afrontamiento enfocado en la emoción) & 2,26 & 1,93 & 0,168 \\
Factor 3 (afrontamiento religiosolespiritual) & 3,64 & 3,43 & 0,959 \\
Factor 4 (búsqueda de apoyo social) & 2,7 & 2,3 & 0,678 \\
\hline
\end{tabular}

EMEP: Escala de modos de afrontamiento de problemas; *Test pareado de Wilcoxon

Tabla 4. Comparación de las variables entre los grupos, Etapa 1 (preintervención)

\begin{tabular}{lccc}
\hline Variable & GE $(\mathbf{n}=\mathbf{8})$ & GC $(\mathbf{n}=\mathbf{1 5})$ & Valor $^{\text {* }}$ \\
\hline Factor 1 (afrontamiento centrado en el problema) & 4,06 & 3,55 & 0,179 \\
Factor 2 (afrontamiento enfocado en la emoción) & 2,53 & 2,26 & 0,349 \\
Factor 3 (afrontamiento religioso/espiritual) & 4,0 & 3,64 & 0,180 \\
Factor 4 (búsqueda de apoyo social) & 2,8 & 2,7 & 0,893 \\
BAl (Ansiedad) & 11 & 12,5 & 0,396 \\
\hline
\end{tabular}

BAI (BECK Anxiety Inventory); GE= grupo experimental; GC= grupo control; *test $\mathrm{U}$ de Mann-Whitney

Tabla 5. Comparación de las variables entre los grupos, Etapa 2 (posintervención)

\begin{tabular}{lccc}
\hline Variable & GE (n=8) & GC (n=15) & Valor $\mathbf{p}^{*}$ \\
\hline Factor 1 (afrontamiento centrado en el problema) & 4,02 & 3,44 & 0,023 \\
Factor 2 (afrontamiento enfocado en la emoción) & 2,17 & 1,93 & 0,182 \\
Factor 3 (afrontamiento religioso/espiritual) & 3,14 & 3,43 & 0,624 \\
Factor 4 (búsqueda de apoyo social) & 2,9 & 2,3 & 0,422 \\
BAI (Ansiedad) & 5,5 & 24 & 0,068 \\
\hline
\end{tabular}

GE= grupo experimental; GC= grupo control; *test $\mathrm{U}$ de Mann-Whitney 


\section{DISCUSIÓN}

La obesidad, considerada una enfermedad crónica, puede ser potencialmente controlada y tener reducción de los efectos adversos desde un tratamiento y orientación adecuados. Esta posibilidad de control puede explicar algunos de los resultados obtenidos mediante la aplicación del EMEP en ambas fases del estudio. Los resultados mostraron que en los pacientes obesos, la estrategia más utilizada es la focalización en el problema, seguido por las prácticas religiosas/ ilusiones, en las dos fases.

La preferencia por estrategias centradas en el problema, independientemente de la exposición a un programa de intervención psicológica, puede deberse a la naturaleza de la responsabilidad individual presente en la obesidad, o a la posibilidad de un mayor control con el seguimiento y la adherencia al tratamiento. Uno puede obtener ayuda y resultados graduales: el seguimiento de un tratamiento y adherencia a las guías, en su caso, son requisitos previos para el éxito potencial y la resolución del problema. Por lo tanto, parece justificado el uso continuado de las estrategias de afrontamiento centradas en el problema como prioridad durante el tratamiento de la obesidad, a diferencia de otras enfermedades, como el cáncer (27), que pueden promover un sentimiento de falta de control sobre el curso de la enfermedad.

En este estudio se ha observado que los pacientes expuestos a la intervención psicológica (GE) aumentaron las puntuaciones en el afrontamiento centrado en el problema y en la búsqueda de apoyo social, así como redujeron las puntuaciones de estrategias centradas en la emoción y en la búsqueda de prácticas religiosas pensamientos fantasiosos/ilusión. Dicho aumento, aunque sin significación estadística, puede sugerir una mayor eficacia en el desarrollo de habilidades de adaptación para hacer frente a la obesidad, el cumplimiento del objetivo del estudio (28).

Por otra parte, los pacientes del grupo de control presentaron una reducción de tres factores (problema, emoción, y la búsqueda de apoyo social), excepto el Factor de 3. Este resultado señala los efectos de la larga espera para el inicio de cualquier tratamiento para la pérdida de peso, como promotor de estrategias no adaptativas que no favorecen cambios en los hábitos alimentarios. Estos hechos parecen reforzar la necesidad de inclusión de pacientes obesos sometidos a tratamiento para la pérdida de peso, en particular, el acompañamiento psicológico, de manera pronta y eficaz.

Esto puede demostrar que adoptando un enfoque de resolución de problemas de naturaleza psicosocial, a medida que la enfermedad se desarrolla, la incontrolabilidad percibida en relación con la obesidad aumenta, y es más difícil identificar y participar en conductas facilitadoras de la pérdida de peso. Por lo tanto, "no hay mucho que hacer" para afrontar activamente a la obesidad y sus consecuencias adversas. Esta condición puede tener resultados perjudiciales, y demuestra la urgencia de intervenciones eficaces de tratamiento tan pronto como sea posible.

Además del aumento en el uso de estrategias no siempre favorables para el GC, también hubo una reducción en las estrategias consideradas eficaces, como las centradas en el problema y en la búsqueda de apoyo social en los participantes. Por lo tanto, la no aplicación de tratamiento psicológico a estos pacientes puede aumentar los efectos colaterales de la obesidad, incluyendo una mayor probabilidad de aislamiento social.

Estos datos señalan una tendencia positiva de la intervención, en términos de aumento de las estrategias más adaptativas. Además, demuestra los daños potenciales de no incluir a pacientes obesos en modalidades de tratamiento tempranos. La exclusión de los pacientes de los programas de intervención psicológica parece reducir las estrategias de afrontamiento consideradas adaptativas, y puede conducir a un mayor riesgo de desarrollar trastornos psicológicos.

Estos datos están en línea con la literatura previa, que señala que la capacidad de resolver problemas (referenciado como estrategias de afrontamiento adecuadas) aumenta en la medida en que las actitudes dietéticas inadecuadas disminuyen, de manera funcionalmente relacionada con una red de apoyo social más satisfactoria (2); o aun, el afrontamiento más activo es considerado cómo predictivo para mejores resultados de pérdida de peso (29). Además, estos resultados corroboran con otros estudios, como lo de Santos ${ }^{(30)}$ que, con metodología similar, encontró que pacientes obesos sometidos a intervención psicológica han desarrollado habilidades de afrontamiento más adaptativas, lo que no pasaba con el grupo control. Sin embargo, estos pacientes no eran candidatos a cirugía bariátrica, enseñando que aún faltan estudios que evalúen estas variables de afrontamiento en esta población específica.

Con relación al grupo de intervención, en términos de puntuaciones generales, no se observaron diferencias significativas entre las dos fases, a pesar de las puntuaciones más bajas después de la intervención. Ya que se esperaba encontrar una mayor reducción de la ansiedad en los pacientes del grupo experimental, algunas hipótesis pueden ser ofrecidas para estos datos: los pacientes tenían, en general, bajos recursos y estaban 
experimentando dificultades que iban mucho más allá de la obesidad. En las sesiones de grupo se informó de diversos problemas familiares como consumo de drogas y alcohol en la familia, conflictos y separaciones matrimoniales, y episodios de violencia doméstica. Además, por problemas administrativos del hospital, se registraron casos de retardo o suspensión de la cirugía, situación que aumentaba la ansiedad de los pacientes.

Sin embargo, aunque con una reducción no satisfactoria en la ansiedad, en comparación con los pacientes sin exposición a la intervención psicológica, se observaron diferencias en los niveles de ansiedad que se pueden considerar marginalmente significativas $(p=0,068)$. Pacientes del grupo de intervención mostraron puntuaciones de ansiedad significativamente más bajos que los pacientes en el GC. Estos datos son relevantes para justificar la necesidad de continuar el asesoramiento psicológico a los pacientes obesos.

Como limitaciones del estudio se tiene la muestra final reducida $(n=18)$ y heterogénea que no permite generalizaciones acerca de los resultados para dimensiones poblacionales y puede haber influido en los resultados obtenidos. Además, al no permitir realizar ANOVA, por ejemplo, no se puede garantizar la fiabilidad de los resultados de la relación entre la condición y el tiempo (tratamiento $x$ control; pre $x$ post). Además, la no realización de una estimación de la muestra es otra limitación y estuvo relacionada al hecho de que esta era de conveniencia ya que estaba directamente asociada a reglas de entrada de pacientes y limitaciones del hospital en que se realizó la investigación.

La necesidad de más tiempo para la realización de la intervención para asegurar el mantenimiento de mayores éxitos, así como la garantía de obtener otras mejoras, tales como la reducción de los niveles de ansiedad en un mayor número de participantes, es otro punto limitante. Así, se puede sugerir que la participación en el programa genera una tendencia de mejora en los pacientes, sin embargo no garantiza efectos causales de estos.
Por todo ello, futuras investigaciones deberían realizarse de forma multicéntrica y con pacientes en acompañamiento continuo (Treatment as usual), de forma que se puedan hacer comparaciones más potentes y llegar a conclusiones sobre la eficacia del programa. En general, se considera ventajoso este protocolo de funcionamiento, ya que se desarrolló en un contexto adverso y aun así se han observado resultados positivos, pero se sugieren las mejorías, como: incluir terapia individual y desarrollar un mejor enfoque para los trastornos de ansiedad. Se destaca que un plan para la futura recolección de datos de seguimiento en el posquirúrgico se está llevando a cabo.

En conclusión, la obesidad es una enfermedad que trae un considerable grado de ansiedad y dificultades para desarrollar estrategias de afrontamiento eficaces no solo para hacer frente a las condiciones adversas, también para el tratamiento necesario. La participación de los pacientes en el Programa de Intervención Psicológica de Preparación para Cirugía Bariátrica demostró ventajas por desarrollar estrategias más eficaces, con una menor carga de sufrimiento, y la no participación presenta efectos potencialmente negativos. Sin embargo, se sugiere nuevas investigaciones, que puedan considerar otros contextos de la salud, así como observar los efectos de la intervención en el largo plazo y con muestras más numerosas.

Agradecimientos: $\mathrm{MM}$ agradece al Conselho Nacional de Desenvolvimento Científico e Psicológico (CNPQ), por la beca de máster que posibilitó la realización de este estudio en la Universidade de Brasília y a la Coordenação de aperfeiçoamento de pessoal de nivel superior (Capes) por la beca de doctorado actual en la Universidad de València.

Contribuciones de los autores: MM y ALCJ han participado en la concepción y diseño del estudio, análisis e interpretación de datos, redacción del artículo, y revisión crítica del artículo. Además, MM ha trabajado en la selección de pacientes y aplicado la intervención en el hospital.

Fuente de financiamiento: este estudio fue autofinanciado.

Conflicto de interés: los autores declaran no tener conflictos de interés en la publicación de este artículo.

\section{REFERENCIAS BIBLIOGRÁFICAS}

1. Harvey SB, Glozier N, Carlton O, Mykletun A, Henderson M, Hotopf $\mathrm{M}$, et al. Obesity and sickness absence: results from the CHAP study. Occup Med (Lond). 2010;60(5): 362-8. doi: $10.1093 /$ occmed/kqq031

2. Keskin G, Engin E, Dulgerler S. Eating attitude in obese patients: the evaluation in terms of relational factors. J Psychiatr Ment Health Nurs. 2010;17(10): 900-908. doi: 10.1111/j.1365-2850.2010.01608.x

3. Costa FS, Bandeira DR, Trentini C, Brilmann M, Friedman R, Nunes MA. Considerações acerca da avaliação psicológica das comorbidades psiquiátricas em obesos. Psicol Estud. 2009; 14(2): 287-93. doi: 10.1590/ S1413-73722009000200009

4. Organización Mundial de Salud(OMS) Health Topics: obesity [Internet]. Ginebra: OMS; 2014 [Citado el 02 de marzo de 2014]. Disponible en: http:// www.who.int/topics/obesity/en/ 
5. Jia H, Lubetkin EI. The impact of obesity on health-related quality-of life in the general adult US population. J Public Health (Oxf). 2005;27(2):15664. doi:10.1093/pubmed/fdi025

6. Greenberg I, Perna F, Kaplan M, Sullivan MA. Behavioral and psychological factors in the assessment and treatment of obesity surgery patients. Obes Res. 2005;13(2): 244-9. doi: $10.1038 /$ oby.2005.33

7. Gade H, Hjelmesaeth J, Rosenvinge JH, Friborg O. Effectiveness of a cognitive behavioral therapy for dysfunctional eating among patients admitted for bariatric surgery: a randomized controlled trial. J Obes. 2014; 127936. doi: $10.1155 / 2014 / 127936$

8. Travado L, Pires R, Martins V, Ventura C, Cunha S. Abordagem psicológica da obesidade mórbida: Caracterização e apresentação do protocolo de avaliação psicológica. Aná Psicológica. 2004; 22(3): 533-50. doi: 10.14417/ap.225

9. White JR. Introdução. En: White JR, Freeman AS, editores. Terapia cognitivo-comportamental em grupo para populações e problemas específicos. São Paulo: Roca; 2003; p. 109-138.

10. Weineland S, Arvidsson D, Kakoulidis T, Dahl J. Acceptance and commitment therapy for bariatric surgery patients, a pilot RCT. Obes Res Clin Pract. 2012;6(1):e1-e90. doi: 10.1016/j. orcp.2011.04.004

11. Tayyem R, Ali A, Atkinson J, Martin CR. Analysis of health-related qualityof-life instruments measuring the impact of bariatric surgery: systematic review of the instruments used and their content validity. Patient. 2011; 4(2): 73-87. doi: 10.2165/11584660000000000-00000

12. Ogden J, Hollywood A, Pring C. The impact of psychological support on weight loss post weight loss surgery: a randomised control trial. Obesity Surgery. 2015;25(3):500-5. doi: $10.1007 / \mathrm{s} 11695-014-1428-2$

13. Hörchner R, Tuinebreijer WE, Kelder H, Urk E. Coping behavior and loneliness among obese patients. Obes Surg. 2002;12(6):864-8. doi:

\subsection{1/096089202320995718}

14. Li W, Rukavina P. A review of coping mechanisms against obesity bias in physical activity/education settings. Obes Rev. 2009;10(1):87-95. doi: 10.1111/j.1467-789X.2008.00528.x

15. Antoniazzi AS, Dell'Aglio DD, Bandeira DR. O conceito de coping: uma revisão teórica. Estud psicol (Natal). 1998; 3(2):273-94. doi: 10.1590/S1413-294X1998000200006

16. Folkman S, Lazarus RS. An analysis of coping in middle-aged community sample. J Health Social Behav. 1980;21(3):219-39.

17. Conradt M, Dierk JM, Schlumberger P, Rauh E, Hebebrand J, Rief W. What copes well? Obesity-related coping ans its associations with shame, guilt and weight loss. J Clin Psychol. 2008;64(10):1129-44. doi: 10.1002/ jclp.20501

18. Puhl R, Brownell KD. Ways of coping with obesity stigma: review and conceptual analysis. Eat Behav. 2003;4(1): 53-78. doi:10.1016/S14710153(02)00096-X

19. Oliveira VM, Linardi RC, Azevedo AP. Cirurgia bariátrica _ aspectos psicológicos e psiquiátricos. Revista de Psiquiatria Clínica. 2004;31(4):199201. doi: 10.1590/S010160832004000400014

20. Rutledge T, Groesz LM, Savu M. Psychiatric factors and weight loss patterns following gastric bypass surgery in a veteran population. Obes Surg. 2011;21(1):29-35. doi: 10.1007/ s11695-009-9923-6

21. Gimenes MGG, Queiroz B. As diferentes fases do enfrentamento durante o primeiro ano após a mastectomia. En: Gimenes MGG, Fávero $\mathrm{MH}$, editores. A mulher e o câncer. Campinas: Editorial Psy; 1997. p. 171-195.

22. Beck AT, Epstein N, Brown G, Steer RA. An inventory for measuring clinical anxiety: psychometric properties. J Consult Clin Psychol. 1988;56(6):893-7. doi: 10.1037/0022006X.56.6.893

23. Cunha JA. Manual da versão em português das escalas Beck. São Paulo: Casa do Psicólogo; 2001.
24. Beck JS. Pense Magro: a dieta definitiva de Beck. Porto Alegre: Artmed; 2008.

25. Saunders R. Post-surgery group therapy for gastric bypass patients. Obes Surg. 2004:14(8):1128-31. doi: $10.1381 / 0960892041975532$

26. Baker RC, Kirschenbaum DS. Selfmonitoring may be necessary for successful weight control. Behav Ther. 1993;24(3):377-394. doi:10.1016/ S0005-7894(05)80212-6

27. Kohlsdorf M, Costa-Junior AL. Estratégias de enfrentamento de pais de crianças em tratamento de câncer. Estud psicol (Campinas). 2008;25(3):417-29. doi: 10.1590/ S0103-166X2008000300010

28. Ortega J, Fernandez-Canet R, Alvarez-Valdeita $S$, Cassinello $\mathrm{N}$, Baguena-Puigcerver M. Predictors of psychological symptoms in morbidly obese patients after gastric bypass surgery. Surg Obes Relat Dis. 2012;8(6): 770-6. doi: 10.1016/j. soard.2011.03.015

29. Figura A, Anhis A, Stengel A, Hofmann T, Elbelt U, Ordemann J, et al. Determinants of Weight Loss following Laparoscopic Sleeve Gastrectomy: The Role of Psychological Burden, Coping Style, and Motivation to Undergo Surgery. J Obes. 2015; 2015: 626010. doi: $10.1155 / 2015 / 626010$

30. Santos PLS. Efeitos de intervenção interdisciplinar em grupo para pessoas com diagnóstico de sobrepeso ou obesidade [tesis para maestría]. Brasil: Departamento de Psicologia Escolar e do Desenvolvimento. Universidad de Brasilia; 2010.

Correspondencia: Marinna Simoes

Mensorio

Dirección: Calle Mestre Buenaventura

Pascual 13,3. 46020. Valencia, España.

Teléfono: +34622366859

Correo electrónico:ninamensorio@gmail.com 